\title{
NON-LINEAR MINI-MAX PROBLEM OF MULTI-STOP FLIGHT ROUTES
}

\author{
Srećko Krile ${ }^{1}$, Marina Krile ${ }^{2}$, Petr Průša ${ }^{3}$ \\ ${ }^{1}$ Dept of Electrical Engineering and Computing, University of Dubrovnik, Croatia \\ ${ }^{2}$ Dept of Mass Communication, University of Dubrovnik, Croatia \\ ${ }^{3}$ Jan Perner Transport Faculty, University of Pardubice, Czech Republic
}

Submitted 26 August 2014; resubmitted 11 November 2014, 8 January 2015; accepted 17 February 2015

\begin{abstract}
The major change in trends of air transport development is to increase operating efficiency, productivity and profitability. Airline companies (air operators or carriers) can ensure significant fuel savings and better utilization of airplanes capacity. So they need better transport planning tool for costs minimization on a voyage route with multiple loading/unloading airports (multi-stop routes). The existing routes can be re-optimized (improved) or alternative routes can be offered instead. In this research the efficient heuristic algorithm for optimal transportation of $N$ different loads (e.g. passenger contingent) for the airplane with limited capacity is being developed. The main goal of algorithm is to reduce the idle capacity on the route, to decrease expenses and to increase revenue. Through many test examples we can see that such approach functions very good and calculation complexity is under control.
\end{abstract}

Keywords: airline industry; passenger transport; multi-stop flight routing; route optimization; route profitability; non-linear mini-max problem.

\section{Introduction}

Today air transport industry is influenced indirectly by the economic recession, increase of fuel cost, stiff competition and political instability. Airline companies (carriers) have a big responsibility to satisfy people's needs and in the end to gain a profit. The most important thing for them is to have efficient routes without empty seats. The major change in trends of air transport development is to increase operating efficiency, productivity and profitability, so more and more routes with multiple stops (landings) are introduced. In other hand the people wants to be transported for the shortest period of time and for the lowest price. The scheduling of multi-stop flight routes is the crucial element and very delicate problem, especially in the relation to airplane capacity, the airport slots, the airplane rental charges, flight clearances and landing permits, airport handling costs, agent's fees and other cost elements and their influence to profit (Barnhart et al. 2009; Carey 2007).

Potential passenger contingents are waiting to be transported with one or more airline carriers, using limited capacity (one or more airplanes). Efficient transfer of passengers between airports is very demanding problem for the carrier (operator). Such problem exists for any direction and for any contingent.
However, the general problem can be seen in two ways. In the first case, the passenger demands are directed to definite carrier (operator) and for existing routes. In that case, the route planning on the operational level has to fulfil all demands (bookings etc.). If demands increase in time, the carrier has to expand transport capacity (bigger airplane or more units) that is very expensive. If demands for some directions are decreasing, the sufficient capacity appears. The routes are firmly defined and cannot be instantly changed. However, potentially, some of point-to-point oriented routes could be switched to multi-stop routes. The space for re-routing on operational level is very small, mostly caused by journey prolongation and restrictions of time scheduling. In general, the route modification (re-routing) includes the new airport on the route or eliminating of the existing airport from the route. In the last option carrier has to ensure the alternative route (another airplane) to fulfil all demands, of course, if it possible in severe limitations in route scheduling. Switching to multi-stop routes could be attractive to passengers only if it ensures significantly lower transport prices (ticket discounts) or in exceptional cases when alternative does not exist.

In the route, planning on strategic level new routes can be introduced or some existing routes can be rerouted. Creation of multi-stop (multi-hop) routes and 
definition of appropriate airplane capacity can be a crucial part of it. The airline operator (carrier) can plan to transport only a part of demands from the potential market (statistically data + forecasting). In other words, the carrier has to decide about the share it wants to participate in transport for each passenger contingent that potentially exists on the market. Of course, it has to be in relation to available capacity (airplane seats).

Introduction of multi-stop routes is in firm correlation to the flight efficiency of each airplane and whole carrier fleet. Therefore, it could be the key for better capacity utilization and transport cost reduction. Multi-stop routes can be very effective; especially in the case of small contingents, that carrier wants to serve. Prolongation of the journey really appears, but it has to be announced in advance. It is less critical factor than expenses nowadays.

In advance this paper deals with the capacity management problem extended to transportation problem of different contingents transported by one transport mean on the route with multiple sources (loading ports) and multiple destinations (ports of discharge). We call it multi-stop (multi-hop) transportation problem. The special case is the point-to-point route where passengers can load the whole airplane without empty seats.

In such strategic planning, we are looking for the set of airports on the route that can ensure the best profit to carrier (operator), in the first case the reduction of transport cost per passenger. In advance all transport elements and costs have to be known: Ticket prices (freight cost) are projected in advance, too. They have to be concurrent on the existing market so carrier has to find space for such cuttings.

For an example the network of airports and distances between them are shown on Fig. 1. Here we have five potential airports and distances between them in $\mathrm{km}$ or miles. Passenger wishes (demands) are shown in respect to transport capacity (in percentage of total airplane capacity that carrier use) (Fig. 2). If transport cost (and profit) depends only of distances it is clear that the airplane will avoid airport 3 and 4, to reduce the transportation cost, as it is shown with dotted line on Fig. 1. The airport 2 has to be on the route because the efficiency is increased with load 2-5. As we can see on Figs 3-4. the airplane is barely empty. However, if we increase flight tickets for some directions e.g. for con-

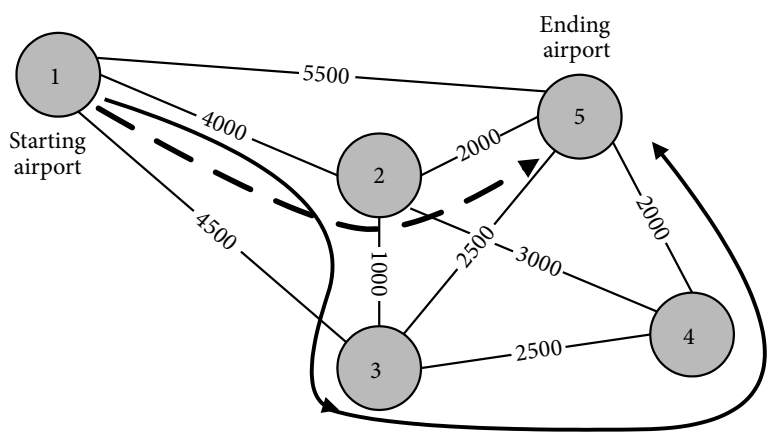

Fig. 1. Distances between airports

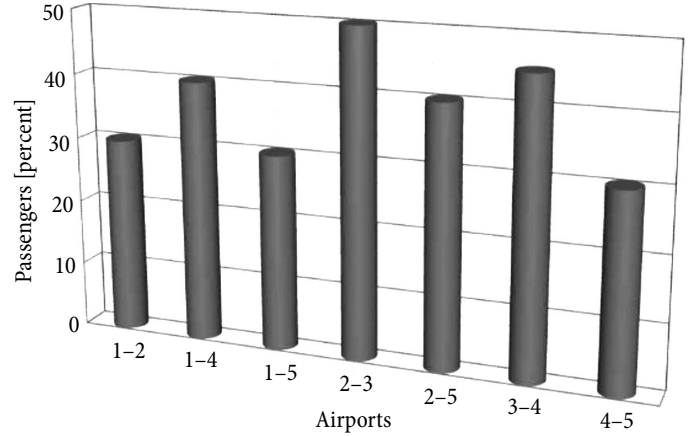

Fig. 2. Potential loads of passengers (contingents) in percent of total airplane capacity

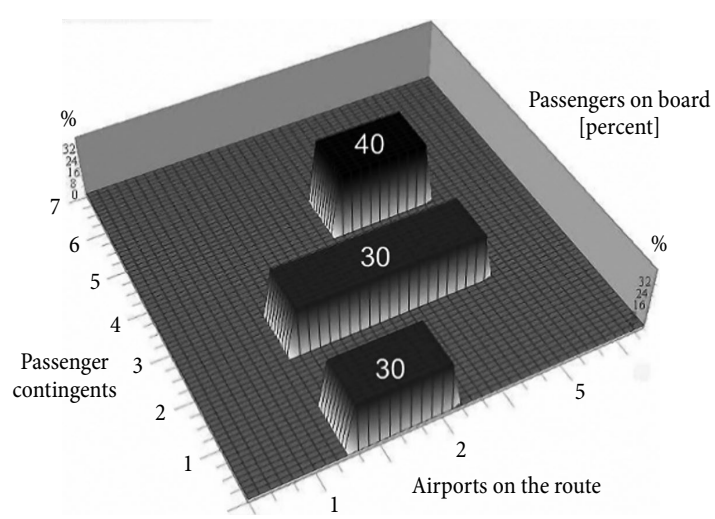

Fig. 3. The optimal route with passengers on board (loads) in percent of total capacity of airplane

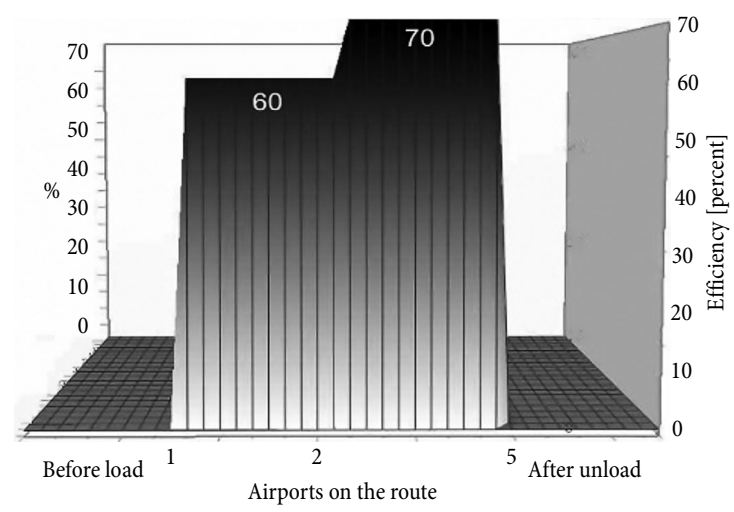

Fig. 4. Efficiency of the route

tingent 3-4 (for only $10 \%$ ) the optimal routing sequence will include the airports 3 and 4, see full line on Fig. 1 . Details are shown on figures in chapter 5.

In the chapter 1 we explained the role of optimization in airline industry through recent years and same important papers with that thematic are mentioned. In the chapter 2 the mathematical model for the routing problem on strategic level is explained. In the chapter 3 the algorithm development is shown. In the chapter 4 we talk about costs and objective function that is nonlinear. In the chapter 5 we can see one test-example and capabilities of algorithm through illustrated results. As the algorithm has some limitations, the algorithm improvements are proposed in the chapter 6. 


\section{Previous Work}

Operational decisions are divided on the route definition, flight frequency, departure time, type of airplane, fleet size with the aim to provide efficient transportation at the lowest possible cost. Complicated process has to be supported by the computers and informatics that are capable to optimize it and find out the appropriate and acceptable solution.

The most important issues to enhance the airline operation efficiency are flight routing and fleet scheduling. Generalized approach to multi-commodity transportation problem we can find in the early paper of Wollmer (1970). Wollmer finds out that capacity of the air corridors are virtually unlimited; however, the number of flight assignments would be constrained by number of planes, pilots, same as with upper bound of seats for defined airplane type (capacity).

Many network flow techniques and models exist to solve the complex mathematical problem in flight routing (Ouorou et al. 2000; Trochim 2006; Vasigh et al. 2008). Model for fleet routes is based on the Multiple Commodity Network Flow Problem (MCNFP) introduce in paper by Yan and Tseng (2002).

Allocation of the expenses and revenue are the basic things that must be considered to evaluate the route profitability. Some costs can be caused directly and some indirectly. These data are very important to determine the correct calculation and profitability of the each route (Chang, Schonfeld 2004).

In fleet routing and multi-stop flight scheduling the crucial elements are the setting the available airplanes, the airport slots, the airplane rental charges, airport service cost (quota), fuel consumption, maintenance cost and other cost elements, which lead to the minimization of all expenses and maximization of the company's profit (Yan, Young 1996).

Short-term flight scheduling model is developed and applied to Taiwan airlines. Such model is defined as a non-linear integer program that is known as NP-hard problem. Non-linear problem is more difficult to solve than the traditional flight-scheduling problem that is defined as integer linear program. The heuristic methods and algorithms can improve such approach significantly (Yan et al. 2007b).

In the paper by Yan and Chen (2002) is developed the model for Taiwan inter-city bus carriers. The model is based on integer multiple commodity network flow problem, too. They solve the problem of bus movements and passenger flows by interconnecting the passenger trip demands on each bus stop and bus capacity defining the time schedule for the route.

In the literature, many papers have been already devoted to ship routing in marine industry. Ferry fleet routing problem is solved by time space network technique that is specified to the defined time period (one day in this paper). In that technique represented by network structure, horizontal axis symbolizes airport locations and vertical axis represents the time duration (distance). Each arc between airports represents activity of ferry transport (Yan et al. 2007a).
Another group articles are concerned by vehicle routing problems. In the paper of Garaix et al. (2010) the optimization of routing vehicles in freight or passenger transport is presented. In that, approach the road network is represented as weighted complete graph. Each arc stands for the shortest route for a potential pair of stops. One arc can be determined by numerous characteristic like travel duration, travel cost, etc. With the consideration of alternatives routes, they evaluate an impact on solution algorithms and values through the graph of the road network. During this representation for vehicle routing problem the fixed sequence arc selection problem is raised. They proposed a dynamic programming solution method for solving that problem. The base element in such approach is to determine the shortest path with resource constraints like vehicle transportation capacity and maximal travel duration. Alternative paths offer a compromise between these components but some of them cannot comply with defined constraints and have to be eliminated from optimal solution.

In the article written by Stojković et al. (2002), DAYOPS (DAY of OPerations Scheduling) model is presented. Every arc presents each flight leg, which means a distance between departure and arrival. Additional arcs present maintenance and ground service transfer between airplane and rest, passenger connections, and crew movements. In this research, four phases of planning process are presented and includes flight scheduling, fleet assignment, airplane routing, and crew scheduling. DAYOPS model can be applicable as fifth phase when these four phases are completed. Model can be used to re-optimize the route schedule at the high level and at the lower level. At the higher level, some flights can be cancelled or at the lower level, the model will find out a new best and profitable flight route.

The load factors, airline frequency, airplane size are necessary issues that must be taken in consideration for making the airline profitable. Choosing an appropriate airplane size for the flight route must be appropriate to the level of demand. This factor can influence a lot on the optimization of the flight route and optimum load occupancy of the airplane (Givoni, Rietveld 2006).

To determine the profitability of the route some operating cost must be verified. These costs are fixed direct operating costs, variable direct operating costs, and indirect costs. Maintenance cost, airplane rental, fuel and oil per airplane, airport services cost have an influence on the revenue and finally on the profitability of the flight. Maintenance costs include engine repair, consumption parts for airplane, technical support, technical documentation, maintenance staff, and other maintenance costs (Gomm 2005).

Airport service cost include landing cost and handling cost for airplane, transhipment of passengers, gasoline, luggage and freight on the airport. Each airport determines the cost for using the airport for landing and handling their airplanes (Tatalović et al. 2009).

Many carriers installed the route planning software with the goal to optimize their existing routes, to increase profit and decrease expenses. With such systems, 
we can clearly see the picture of the costs that influence on the route profitability and the way how to improve it. In addition, the software helps the pilots to find a better balance of fuel usage, flight speed and flight path. The efficiency today is the most important element and the costs must be minimized wherever it is possible. Such optimization tool could be the crucial thing in any intelligent transportation and it influences on the airline profitability significantly.

\section{Mathematical Model}

One of the most important problems in airline transportation is to find the sequence of passenger distribution between multiple sources and multiple destinations (stops), minimizing the transportation cost and looking for better utilization of the airplane capacity.

Amounts of different passenger contingents are in firm correlation because the total capacity of airplane is limited. Taking into account passenger demands for each airport and each destination (sufficient amount of passengers waiting to be transported), we need optimal transportation plan to minimize shipping and loading/ unloading expenses, transportation cost and cost of airport costs (connected with expenses at airport handling and loading process). It can help in definition of optimal airplane capacity arrangement or for evaluate the route efficiency. The problem of optimal transportation from multiple (several) airports of loading (sources) to multiple destinations (sinks) is very hard (NP-hard) optimization (combinatorial) problem (Wollmer 1970).

Different passenger contingents are differentiated with $i$ for $i=1,2, \ldots, N$. The plane with defined capacity is shipping from the first to the last airport marked with $M+1$, with possible set of intermediate ports marked with $K$. The objective is to find a loading and transportation strategy that minimizes the total cost incurred over the whole voyage route consisting of $M$ airports on the path $(M \leq K)$. We need the loading plan for various passenger contingents waiting in each airport, so the route will be capable to serve the $N$ passenger loads from loading airport to destinations (landing point). The starting airport on the route can be only for loading and the last airport on the route can be only for unloading; other airports on the route may be for both. We can change the starting and the ending point, too. That case is more appropriate in definition of new flight routes.

The transportation technique explained above can be seen as the Capacity Expansion Problem (CEP). Transmission portions of the airplane space are capable to serve $N$ different passenger loads (multi-commodity) for $i=1,2, \ldots, N$. For each passenger load, we need a part of airplane capacity, so it looks like CEP on the path, from zero to maximum (Castro, Nabona 1996).

New capacity portion on the board of aircraft can be assigned to appropriate passenger load up to the given limit (maximal capacity). Used capacity can be dimensioned in two forms: by expansion or by reduction. Expansions/reductions can be done separately for each passenger contingent (load). Fig. 2 gives an example of network flow representation for multiple contingents
$N$ and $M$ airports on the route. So, the transportation problem can be represented by a flow diagram of oriented acyclic network.

Let $G(V, E)$ denote a network topology, where $V$ is the set of vertices/nodes, representing capacity states on the board and $A$, the set of arcs (links) representing traffic changes (loading/unloading, transportation, airport services etc.) between airports. Each link on the route (path) is characterized by $z$-dimensional link weight vector, consisting of $z$-nonnegative weights (Fleischer 2000). In general we have Multi-Constrained Problem (MCP) with multi-dimensional link weight vectors for $M+1$ links on the path $\left\{w_{i, m}, m \in A, i=1\right.$, ..., $N\}$. The constraints for capacity bounds are denoted with $L_{i, m}\left(L_{1, m}, L_{2, m}, \ldots, L_{N, m}\right)$. For an additive measure (load of passengers) definition of the constrained problem is to find a path from the starting to the end airport with minimal weight to satisfy maximal traffic load. It is equivalent with minimal cost that is the function of all expenses. Shorter distance gives lower weight (Fonoberova, Lozovanu 2004). In addition, the weight of each link corresponds to the amount of used capacity. In addition, more people on board causes lower transportation cost of one passenger. The objective is to find the optimal routing/loading sequence that minimizes the total cost with maximal passengers on-board. In the context of MCP we can easily introduce the adding constraints e.g. maximal length of the route.

In the mathematical model of CEP the following notation is used:

$-i, j$ and $k-$ indices for passenger load. The $N$ facilities are not ranked, just present different types of passenger contingents from $1,2, \ldots, N$.

- $m$ - indices the airport of boarding and landing. The number of air of calls on the route including departure airport $M(m=1, \ldots, M)$.

$-u, v$ - indices for airports in sub-problem, $1 \leq u$, ..., $v \leq M$. All airports on the route are transhipment ports except 1 and $M$.

- $x_{i, m}$ - quantity of $i$-th load (e.g. passenger contingent) being loaded on board in airport $m$. Total loading amount in airport $m$ :

$$
X_{m}=\sum_{i=1}^{N} x_{i, m} \text {. }
$$

- $L x_{i, m}$ - capacity limitation for each airport and for each contingent. For convenience, the $x_{i, m}$ is assumed to be integer. For multi-stop routing we suppose that amount of passengers that can be loaded in airport is much lower than total airplane capacity. In opposite the point-to-point routing is more appropriate.

- $r_{i, m}$ - unloading of passengers $i$-th contingent in airport $\mathrm{m}$. For convenience, the $r_{i, m}$ is assumed to be integer. All unloading demands must be satisfied after unloading in last airport on the route. Total unloading amount in the airport $m$ :

$R_{m}=\sum_{i=1}^{N} r_{i, m}$ 
- $I_{i, m}$ - the total amount of passengers transported from airport $m$ to $m+1$. The amount of passenger load $i$ at departure from airport $m$ is equivalent to arrival at airport $m+1$. Before the first airport of loading, $I_{i, 1}=0$. After last airport $I_{i, M+1}=0$ for $i=1, \ldots, N$. Before the first airport of loading, $I_{i, m}=0$. After last airport $I_{i, M+1}=0$ for $i=1, \ldots, N$. Capacity values cannot be negative.

$I_{m+1, i}=I_{m, i}+\sum_{i=1}^{N} x_{i, m}-r_{i, m}$,

for $i=1, \ldots, N ; m=1, \ldots, M$.

- step $I_{i}$ - the lowest step of possible capacity loading and unloading for capacity type $i$. In numerical examples it can be set e.g. step $I_{i}=10 \%$ of total capacity of the airplane.

$-z_{m}$ - the total loading/unloading amount for all types of contingents in airport $m$, related to calculation of airport taxes.

- lon $_{m}$ - maximal length of the each hop, not to exceed the length of the whole route LON.

$z_{m}=\sum_{i=1}^{N} x_{i, m}+r_{i, m}$

$W_{m}=\sum_{i=1}^{N} W_{i, m}-$ airplane transport capacity;

$I_{m}=\sum_{i=1}^{N} I_{i, m}-$ used capacity between airports; $m$ and $m+1$;

$e_{m}=\frac{I_{m}}{W_{m}}-$ the shipping efficiency between airport $m$ and airport $m+1$;

$I D L E_{m}=W_{m}-I_{m}-$ unused airplane capacity after loading/unloading in airport $m$.

\section{Algorithm Development}

Instead of a non-linear convex (polynomial) optimization (Garaix et al. 2010), that can be very complicated and time-consuming, the network optimization methodology is efficiently applied here (Fig. 5). In addition, many different techniques exist, e.g. minimum cost flowbased genetic algorithm (Xie, Jia 2012).

The main reason for our approach is the possibility of discrete capacity values for limited number of contingent loads, so the optimization process can be significantly improved. The multi-constrained routing can be formulated as minimum cost multi-commodity flow problem. Such problem (NP-complete) can be easily represented by multi-commodity the single (common) source multiple destination network.

Definition of the single-constrained problem is to find a path $P$ from starting to end airport such that:

$$
w(P)=\min \sum_{m=1}^{M+1} \sum_{i=1}^{N} w_{i, m}\left(I_{i, m}, x_{i, m}, r_{i, m}\right),
$$

where: $I_{i, m} \leq L_{i, m}$,

satisfying condition:

max distance of $P=\sum_{m_{1}}^{m_{2}} \operatorname{lon}_{i} \leq L O N$,

for $i=1, \ldots, N ; m=1, \ldots, M$.

A path obeying the above conditions is said to be feasible. Note that there may be multiple feasible paths between starting and ending airport (node).

Generalizing the concept of the capacity states after loading/unloading each passenger contingent (load) $m$ between airports on the route we define as a capacity point $-\alpha_{m}$ :

$$
\begin{aligned}
& a_{m}=\left(I_{1, m}, I_{2, m}, \ldots, I_{N, m}\right) ; \\
& a_{1}=a_{M+1}=(0,0, \ldots, 0) .
\end{aligned}
$$

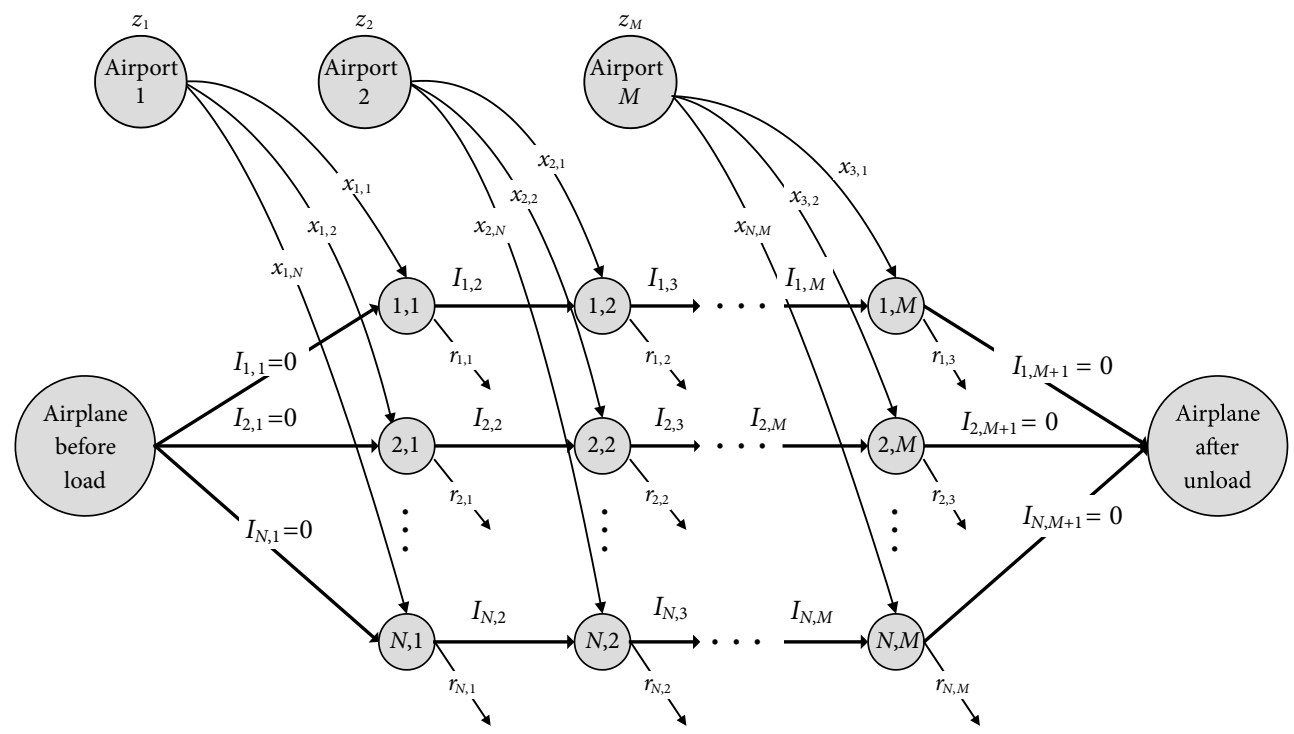

Fig. 5. Transportation problem can be represented by a flow diagram of oriented acyclic network 
In formulation (3.4) $\alpha_{m}$ denotes the vector of capacities $I_{i, m}$ for each load $i$ and for each airport $m$, and we call it capacity point. On the flow diagrams (Fig. 5) each column represents a capacity point, consisting of $N$ capacity state values (for $i$-th passenger load). Formulation (3.5) implies that zero values are before loading on the starting point same as after unloading on the ending point. Let $C_{m}$ be the number of possible capacity point values at airport $m$ (passenger load values for each contingent after departure from airport) (Fig. 6). Only one capacity point is for starting and only one for end airport on the route: $C_{1}=C_{M+1}=1$. The total number of capacity points is:

$$
C_{p}=\sum_{m=1}^{M+1} C_{m} .
$$

Horizontal links (branches) are representing capacity flows between two neighbour airports on the route.

The network optimization can be divided in two steps. At the first step the minimal transportation weights $d_{u, v}$ between all pairs of capacity points (neighbour airports on the route) are calculated. It is obvious that in CEP we have to find many cost values $d_{u, v}\left(a_{u}, a_{v+1}\right)$ that emanate two capacity points of neighbour links (common router), from each node $\left(u, a_{u}\right)$ to node $\left(v+1, a_{v+1}\right)$ for $v \geq u$. Calculation of such value is the Capacity Expansion Sub-problem (CES).

The most of the computational effort is spent on computing of the sub-problem values. That number depends on the total number of capacity points, see (3.6). The total number of all possible $d_{u, v}\left(a_{u}, a_{v+1}\right)$ values representing CES between two capacity points is:

$$
N_{d}=\sum_{m=1}^{M} C_{m} \cdot C_{m+1} .
$$

At the second step we are looking for the shortest path in the network with former calculated weights (Fig. 6).
As the number of all possible $d_{u, v}\left(a_{u}, a_{v+1}\right)$ values depends on the total number of capacity points it is very important to reduce that number $C_{p}$ and that can be done through imposing of appropriate capacity bounds or by introduction of adding constraints (e.g. max. shipment delay). Through numerical test-examples we'll see that many loading/unloading solutions cannot be a part of the optimal expansion sequence. It is the way how algorithm can be significantly improved. Therefore, we can obtain the near-optimal result with significant computational savings.

\section{Objective Function Leads to Non-Linear Problem}

The total cost over time includes:

a) Transhipment cost on distance between airports $m$ and $m+1$ :

$c_{m}=C_{m} \frac{d_{m}}{s}$,

where: $C_{m}$ - transportation cost during voyage [per day]; $d_{m}$ - distance [nautical miles or $\mathrm{km}$ ]; $s$ - airplane speed [knots]. Here it is not correlated with number of passengers on board, influence on speed, oil consumption, agent taxes and freight expenses but it could be easily incorporated. In that case, we have non-linear function instead. In our test-examples the constant speed of airplane is incorporated in the value $C_{m}$.

b) Loading/unloading cost in airport $m$ :

$h_{m}=H_{m} \sum_{i=1}^{N}\left(\frac{x_{i, m}+r_{i, m}}{l o_{m}}\right)=\frac{H_{m} z_{m}}{l o_{m}}$,

where: $H_{m}$ - cost of loading/duration of airplane stay at airport $m$ [minutes]; $l o_{m}$ - operation capacity of airport $m$.

c) Freight cost $f_{i, m}$ for shipment of contingent type $i$ is making profit to airlines company. We want to incorporate the maximal revenue with minimization

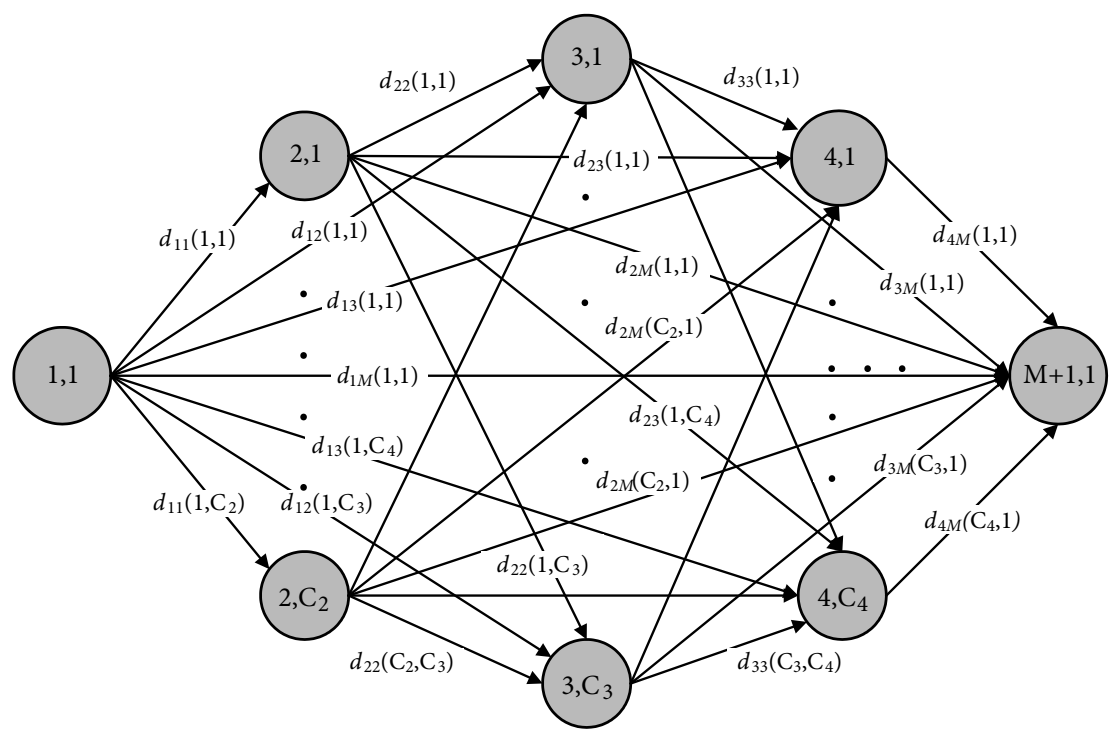

Fig. 6. The CEP problem can be seen as the shortest path problem for an acyclic network in which the nodes represent all possible values of capacity points (the links connecting neighbour airports on the route are representing CES values) 
of expenses within the same optimization process. Therefore, we have to introduce freight cost (airline tickets). We can do that by the exponential cost function showing the economy of scale:

$$
f_{i, m}=A_{i, m}+B_{i, m} \cdot I_{i, m}^{a_{i, m}}
$$

where: $a_{i, m}$ represents the factor of concavity for appropriate contingent type $i$ and for appropriate shipment conditions on the route. The amount of contingent to be transported is in firm correlation with that cost. If we have lower ticket price for some contingent we can expect less passengers on board and vice versa. Optimization process will find out the most attractive cargo plan that is in correlation with all potential contingents waiting to be transported.

The objective function for CEP problem can be formulated as follows:

$\max \left(\sum_{m=1}^{M+1} f_{m}\left(I_{m}\right)-c_{m}\left(d_{m}\right)-h_{m}\left(z_{m}, l_{m}\right)-g_{m}\left(z_{m}\right)-i d l e\left(W, I_{m}\right)\right)$,

so that we have: $I_{i, m+1}=I_{i, m}+D_{i, m}\left(x_{i, m}, r_{i, m}\right)$

$$
D_{i, m}=\sum_{i=1}^{N}\left(x_{k, m}-r_{l, m}\right) ; k \neq l
$$

for $m=1,2, \ldots, M+1 ; k, l=1,2, \ldots, N$.

As we can see from (4.4) the objective function (total cost) includes some different costs. In fact, we have min-max problem. Freight cost (passenger tickets) is denoted with $f_{i, m}\left(I_{i, m}\right)$ and we have to differentiate freight cost for each passenger load (contingent). All expenses have negative polarity. It means that profit will be reduced by transportation cost $c_{i, m}\left(x_{i, m}-r_{i, m}\right)$ and transhipment (load/unload) cost $g_{m}\left(z_{m}\right)$. The airport taxes has to be introduced, too. In $g_{m}$ we can include all airport expenses.

The idle capacity cost idle $\left(W-I_{m}\right)$ could be taken in account, but only as a penalty cost to force the usage of maximal capacity (prevention of unused/idle capacity). In our test-examples it is not included. Costs are often represented by the fix-charge cost or with constant value. It should be assumed that all cost functions are concave and non-decreasing (some of them reflecting economies of scale) and they differ from one airport to another. The objective function is necessarily non-linear and exponential. With different cost parameters the optimization process could be strongly influenced, looking for benefits of the most appropriate transportation solution (Gomm 2005).

Instead of maximization of the profit we can use minimization of the negative value, so in such way we have simplification of very complicated mini-max transportation problem; see (4.4). In both cases, it leads to maximization of the profit.

CES with the similar objective function as (4.4). At second step, we search for the shortest path in the network with former calculated weights between node pairs (capacity points). Suppose that all links (sub-problems) in diagram on Fig. 6 are calculated, the optimal solution for CEP can be found by searching for the optimal sequence of capacity points and their associated link state values. It is well-known shortest path problem for an acyclic network in which the nodes represent all possible values of capacity points. Then Dijkstra's or Floyd's algorithm or any similar algorithm can be applied (Krile 2004, 2005, 2011; Zangwill 1968).

As we said before, the network optimization is divided in two steps. At first step the minimal transportation weights $d_{u, v}$ are calculated between all pairs of capacity points (neighbour airports on the route). The calculation of each weight value between any couple of capacity points has been named: capacity.

For every CES many different solutions can be derived depending on $D_{i}$ values. Each of them represents the capacity state of each contingent on-board the airplane with loading and unloading values (amounts) in appropriate airport.

The complexity of the proposed algorithm is $O\left(C_{p}{ }^{2}\right)$. As we said before $C_{p}$ is in a strong correlation with number of ports $M$ and number of contingents $N$ but also with capacity increment step $I_{i}$ that can be variable from contingent to contingent. We have the problem of large number of capacity states. To simplify the problem we use contingent amounts given in the percentage of the total airplane capacity (Fig. 2).

In our optimization process the number of passengers on board does not influence on voyage speed neither to oil (gasoline) consumption but it could be easily incorporated. The loading strategy consists of loading/ unloading plan for each airport and for each contingent. The starting airport on the route can be only for loading purpose and the last airport is only for unloading; other transhipment airports may be for both.

Some limitations on the capacity can exist, but today the most airports have loads under the airplane capacity.

\section{Results of Trivial Approach}

Here we discus strategic planning for the numerical testexample from Figs 1-2. In the new route definition for test-example, we have starting airport 1 and ending airport 5 , but any of three middle airports can be included in the route.

From Fig. 2 we can see traffic demands (possible transfer of contingents) given in the percentage of the total airplane capacity. That information is gathered through market research or from statistics. From input data we can see seven contingents waiting for transport. It is obvious that the potential loads that can be transported on relations: $1-2(30 \%), 1-4(40 \%), 1-5(30 \%)$, 2-3 (50\%), 2-5 (40\%), 3-4 (45\%) and 4-5 (30\%), but e.g. we have no demands from airport 2 to airport 4 . For simplicity all costs elements are equal $\left(A_{i, m}=0\right.$, $B_{i, m}=10.0 / \%$ of capacity; $C_{i, m}=0.1 / \mathrm{km} ; H_{i, m}=2.0 / \%$ of capacity; $G_{i, m}=0$; and concavity for all costs $a_{i, m}=0.85$. In addition, we have no limitation on flight duration. For the next test-example we slightly changed ticket price (freight cost) only for contingent $6(3-4)$, so $B_{6, m}=12.0$ instead of 10.0. In such example, we want to force travel- 
ling across airports 3 and 4 . According to revenue and all transport costs (transportation price, oil consumption, airport transhipment cost, airport taxes etc.) we can design the route which will be the most profitable. In Figs 7-8 the resulting (the best) route is presented. Fig. 7 shows passengers of each contingent on board the airplane during the voyage. The Fig. 8 shows the loading and unloading amounts of every contingent in particular airport on the route. Now the profit 48.94 is significantly higher than 36.61 from beginning (Figs 2-4).

For the trivial option, we used the same capacity increment step $I_{i}$ for all contingents and it is $10 \%$. We know such capacity resolution is not satisfactorily and, in general, we should be far away for optimal result. In that case, we have 1455 capacity states and $1455 \times 1455$ CES values. For our test-example the best routing option is from airport 1 to airport 2, to airport 3, to airport 4 and finally to airport 5 . The solution does not extract the airports 3 and 4 as before, because it is more profitable to go this way (in spite of longer distance).

Fig. 9 presents the efficiency of the route. Idle capacity during the voyage is obvious but it is lower than before (Fig. 4). Only on the path from airport 3 to 4 we have no idle capacity (100\%). For this example the costs element are similar but it can be differentiated from airport to airport and from contingent to contingent. Low transport efficiency between airports $2-3$ is caused of passenger contingents going between airports 1-4 and $1-5$, that is more profitable (higher distance) than transport from airports $2-3$.

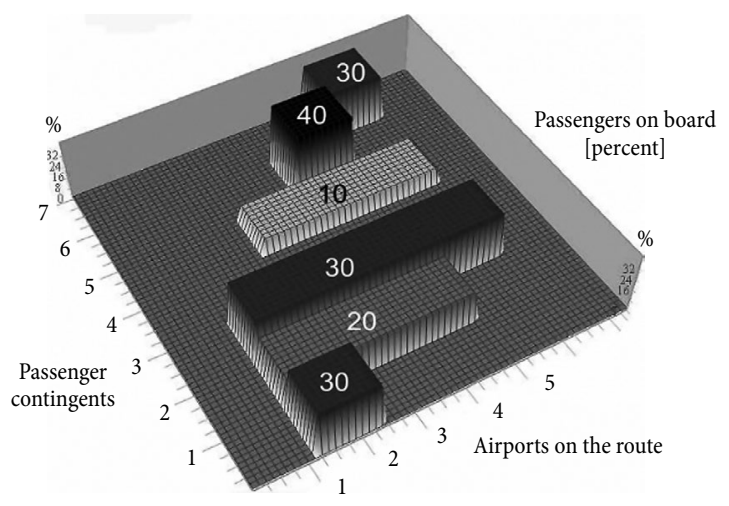

Fig. 7. Airplane's occupancy on the route for each passenger contingent

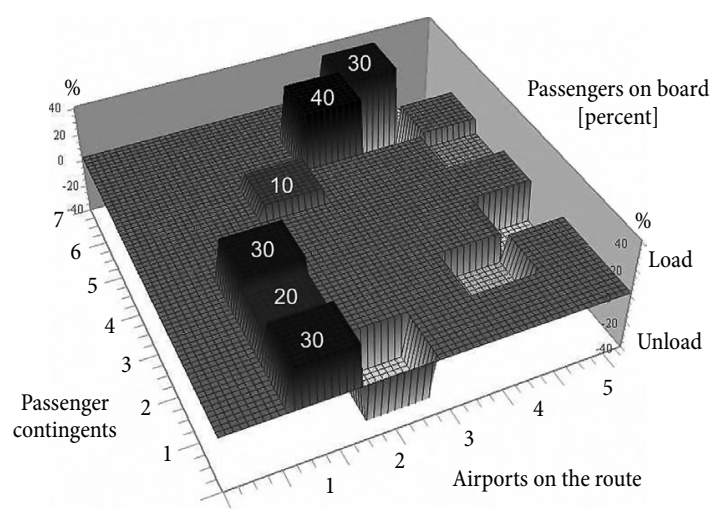

Fig. 8. Loads and unloads in each airport on the route

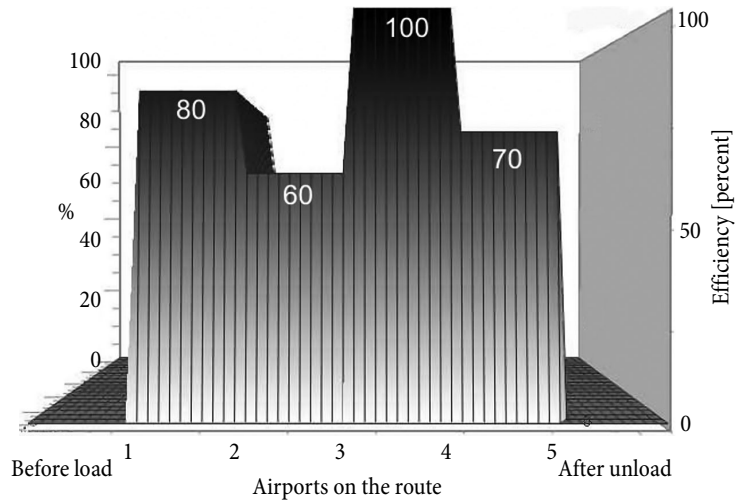

Fig. 9. Efficiency of airplane capacity on the route

\section{Algorithm Limitations and Possible Improvement}

As we said before the crucial element is the number of capacity points $C_{p}$. In previous numerical test-example the starting capacity increment step $I_{i}$ was $10 \%$ and the number of capacity points was 1455 . Calculation of so many CES values; see (3.7) could be very demanding but it is still acceptable.

If we decide to have smaller capacity increment e.g. step $I_{i}=5 \%$ or step $I_{i}=1 \%$ the number of capacity points drastically raises, e.g. it could be more than $10000 \times 10000$ CES values and we need super computer. Therefore, we can shorten the range of capacity points using some techniques of artificial intelligence. For example, the calculated routing sequence defines that the second contingent is in amount of $20 \%$ so we can use the range from $15-25 \%$ only, it means Delta $= \pm 5 \%$. In that case we have significantly less capacity points $C_{p}=$ 3786. The complexity is still acceptable (Table). From Figs 10 and 11 we can see the routing sequence differs from previous result and the profit is slightly raising. From Fig. 12 it is obvious that the idle capacity is still on the similar level, but, in fact, we transported more passengers with profit of 55.76 .

In the next step we can apply the smaller range of capacity e.g. for contingent five we can use $\pm 3 \%$ e.g. $0-6 \%$ of airplane capacity only. In addition, we can use smaller increment step $I_{i}=1 \%$ (that is connected with airplane type and number of seats). Number of CES values increase on 5821 but now we are very close to optimal result (maximal profit). In that case we have profit $=56.66$. With this step-by-step method we can significantly increase the resolution of the capacity states and because of that we can be much closer to the optimum. Of course, we can decrease step $I_{i}$ to smaller value e.g. $0.5 \%$ or less, but it has to be an acceptable value. For example: if airplane has 200 seats step $I_{i}=0.5 \%$ means 1 (one) passenger.

Through many test examples it is clear that such approach functions good and calculation complexity of the optimization process is under control. Without stepby-step calculation the complexity of such approach may be too big. 


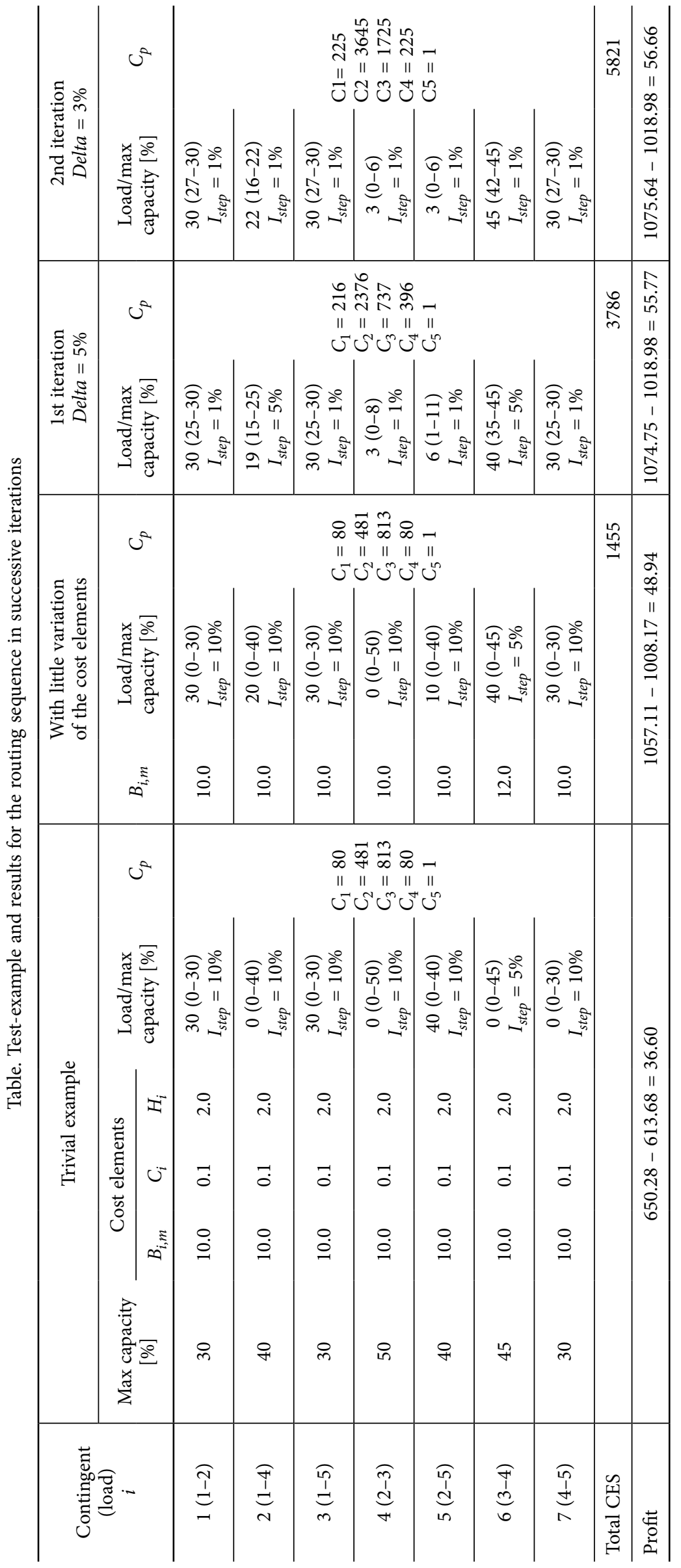




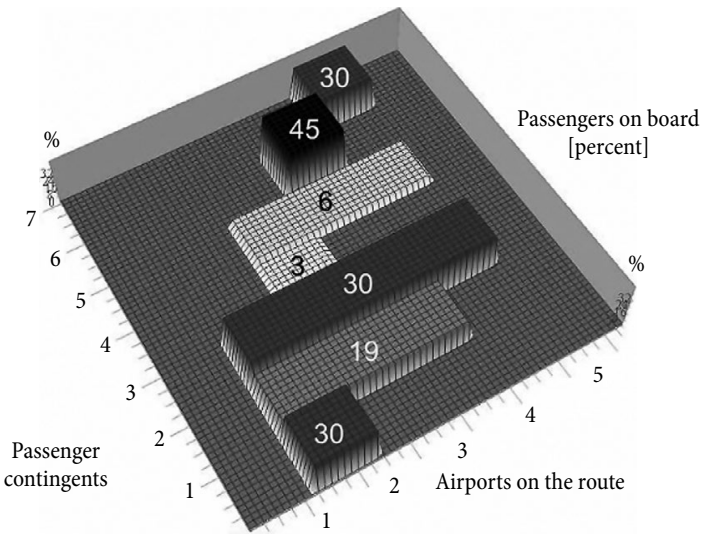

Fig. 10. Airplane's occupancy on the route for the first iteration

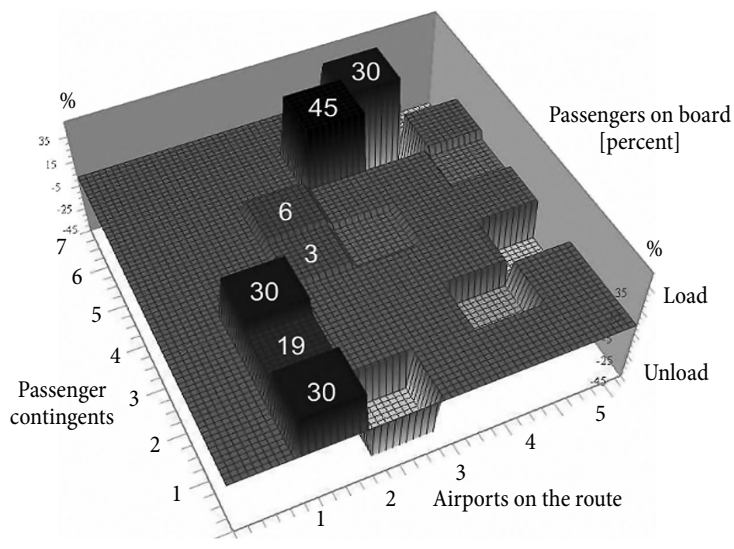

Fig. 11. Loads and unloads in particular airport

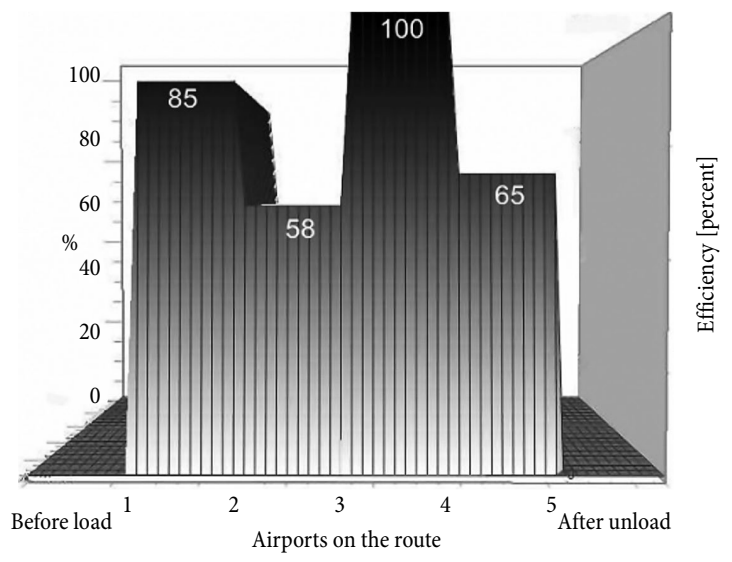

Fig. 12. The efficiency is similar but profit increased

(the strong influence has the contingent 6 (3-4) that is more valuable than others)

\section{Conclusions}

Optimizing the existing flight routes (re-routing) or introducing the new one is the crucial part of strategic planning in airline industry. The transportation problem is extended to capacity management problem of different passenger contingents transported by one airplane on the route with multiple loadings points (boarding) and multiple unloading points (landing destinations).
The proposed heuristic algorithm shows ability to solve very complex non-linear transportation problem. This approach consisting of successive iterations decreases complexity to acceptable level. In the same time, it ensures to airline operators (carriers) very fine modulation of many input values, leading optimization process in wanted direction.

Multi-stop (multi-hop) routes can be more profitable than point-to-point oriented routing. Such optimization tool can create such routes and can help in sizing of appropriate airplane. In addition, the carrier can check the efficiency in opposite direction of the route. With smaller airplanes, the lower number of passengers can be transported if the demand for that particular returning flight is not so high. With comparing the data from both directions, the most efficient routes could be created. Another one possibility in route definition is the change of starting or ending airport, but it is appropriate only in definition of new routes.

\section{References}

Barnhart, C.; Marla, L.; Jiang, H. 2009. Optimization approaches to airline industry challenges: airline schedule planning and recovery, in Dagstuhl Seminar 09261: Models and Algorithms for Optimization in Logistics, 21-26 June 2009, Dagstuhl, Germany, 1-3. Available from Internet: http://drops.dagstuhl.de/opus/volltexte/2009/2188

Carey, S. 2007. Calculating costs in the clouds: how flightplanning software helps airlines balance fuel, distance, wind, 'overfly' fees, The Wall Street Journal, 6 March 2007. Available from Internet: http://www.wsj.com/articles/ SB117314795095227844

Castro, J.; Nabona, N. 1996. An implementation of linear and nonlinear multicommodity network flows, European Journal of Operational Research 92(1): 37-53. http://dx.doi.org/10.1016/0377-2217(95)00137-9

Chang, S.-W.; Schonfeld, P. 2004. Optimized schedules for airline routes, Journal of Transportation Engineering 130(4): 412-418.

http://dx.doi.org/10.1061/(ASCE)0733-947X(2004)130:4(412)

Fleischer, L. K. 2000. Approximating fractional multicommodity flow independent of the number of commodities, SIAM Journal on Discrete Mathematics 13(4): 505-520. http://dx.doi.org/10.1137/S0895480199355754

Fonoberova, M.; Lozovanu, D. 2004. The maximum flow in dynamic networks, Computer Science Journal of Moldova 12(3): 387-396.

Garaix, T.; Artigues, C.; Feillet, D.; Josselin, D. 2010. Vehicle routing problems with alternative paths: an application to on-demand transportation, European Journal of Operational Research 204(1): 62-75. http://dx.doi.org/10.1016/j.ejor.2009.10.002

Givoni, M.; Rietveld, P. 2006. Choice of Aircraft Size - Explanations and Implications. Tinbergen Institute Discussion Paper TI 2006-113/3. 18 p. Available from Internet: http://papers.tinbergen.nl/06113.pdf

Gomm, K. 2005. Predictive planning aids route profitability at BA, Computer Weekly 4/26/2005, 8-8.

Krile, S. 2011. Logistic support for loading/unloading in shipping with multiple ports, in Proceedings of the 31st International Conference of Automation in Transportation, 2011, Pula - Milan, 94-97. 
Krile, S. 2005. Optimal voyage planning in container shipping, in Zbornik radova: 25. skup o prometnim sustavima $s$ medunarodnim sudjelovanjem Automatizacija u prometu 2005 - Proceedings: 25th Conference on Transportation Systems with International Participation Automation in Transportation 2005, 7-12 November 2005, Zagreb/Copenhagen, 32-35.

Krile, S. 2004. Application of the minimum cost flow problem in container shipping, in Proceedings ELMAR-2004: 46th International Symposium Electronics in Marine, 16-18 June 2004, Zadar, Croatia, 466-471.

Ouorou, A.; Mahey, P.; Vial, J.-Ph. 2000. A survey of algorithms for convex multicommodity flow problems, Management Science 46(1): 126-147. http://dx.doi.org/10.1287/mnsc.46.1.126.15132

Stojković, G.; Soumis, F.; Desrosiers, J.; Solomon, M. M. 2002. An optimization model for a real-time flight scheduling problem, Transportation Research Part A: Policy and Practice 36(9): 779-788. http://dx.doi.org/10.1016/S0965-8564(01)00039-8

Tatalović, M.; Babić, Š. R.; Bajić, J. 2009. Airline route profitability modelling, in 12. mednarodno posvetovanje o prometni znanosti - 12th International Conference on Transport Science ICTS 2009, 4-5 June 2009, Portorož, Slovenia.

Trochim, W. M. K. 2006. Research Methods: Knowledge Base. Available from Internet: http://www.socialresearchmethods.net/kb

Vasigh, B.; Fleming, K.; Tacker, T. 2008. Introduction to Air Transport Economics: from Theory to Applications. Ashgate. $382 \mathrm{p}$.

Wollmer, R. 1970. Multicommodity Supply and Transportation Networks with Resource Constraints: the Generalized Multicommodity Flow Problem. Research Memoranda RM-6143-PR. The Rand Corporation, Santa Monica, CA. 40 p. Available from Internet: http://www.rand.org/pubs/ research_memoranda/RM6143.html

Xie, F.; Jia, R. 2012. Nonlinear fixed charge transportation problem by minimum cost flow-based genetic algorithm, Computers \& Industrial Engineering 63(4): 763-778. http://dx.doi.org/10.1016/j.cie.2012.04.016

Yan, S.; Chen, H.-L. 2002. A scheduling model and a solution algorithm for inter-city bus carriers, Transportation Research Part A: Policy and Practice 36(9): 805-825. http://dx.doi.org/10.1016/S0965-8564(01)00041-6

Yan, S.; Chen, C.-H.; Chen, H.-Y.; Lou, T.-C. 2007a. Optimal scheduling models for ferry companies under alliances, Journal of Marine Science and Technology 15(1): 53-66.

Yan, S.; Tang, C.-H.; Lee, M.-C. 2007b. A flight scheduling model for Taiwan airlines under market competitions, Omega: the International Journal of Management Science 35(1): 61-74. http://dx.doi.org/10.1016/j.omega.2005.03.002

Yan, S.; Tseng, C.-H. 2002. A passenger demand model for airline flight scheduling and fleet routing, Computers \& Operations Research 29(11): 1559-1581.

http://dx.doi.org/10.1016/S0305-0548(01)00046-6

Yan, S.; Young, H.-F. 1996. A decision support framework for multi-fleet routing and multi-stop flight scheduling, Transportation Research Part A: Policy and Practice 30(5): 379-398. http://dx.doi.org/10.1016/0965-8564(95)00029-1

Zangwill, W. I. 1968. Minimum concave cost flows in certain networks, Management Science 14(7): 429-450.

http://dx.doi.org/10.1287/mnsc.14.7.429 